\title{
EVOLUCIÓN DE LA AUTOPERCEPCIÓN DEL NIVEL DE ADQUISICIÓN DE COMPETENCIAS DE LOS ESTUDIANTES DE UNA ASIGNATURA DE GRADO EN INGENIERÍA
}

Evolution of self-perception of level of acquisition of competence of students from a course of degree in engineering

Evolução da autopercepção do nível de aquisição de competência de estudantes de umadisciplina de licenciatura em engenharia

Plan de Apoyo a la Innovación Docente 2016 de la Universidad de León (GID 09)

\author{
María-Inmaculada González-Alonso (1) \\ Ramón-Ángel Fernández-Díaz (2) \\ Miguel de Simón-Martín (3)
}

(1) Universidad de León, España. Teléfono: +34 987291765. Correo electrónico: inmaculada.gonzalez@unileon.es

(2) Universidad de León, España. Teléfono: +34 987291761. Correo electrónico: ramon.fernandez@unileon.es

(3) Universidad de León, España. Teléfono: +34 987291000 + 5391. Correo electrónico: miguel.simon@unileon.es

\begin{abstract}
Resumen
El enfoque de la educación en el ámbito universitario y en el no universitario en el que nos encontramos actualmente hace necesario el cambio en las metodologías y dinámicas desarrolladas por los docentes en las aulas. Este trabajo surge de la necesidad de aplicar metodologías activas(De Miguel Díaz et al., 2006) a la formación de los estudiantes universitarios, así como de la búsqueda de la funcionalidad y motivación en el aprendizaje, disponiendo de instrumentos y herramientas con las que el estudiante toma conciencia de su propio proceso de enseñanza-aprendizaje y se fomenta entre ellos el espíritu crítico. La motivación de emprender esta práctica viene suscitada por la inquietud de proporcionar al estudiante capacidades que le ayuden a su desarrollo profesional. En el presente artículo, se pretende constatar en qué medida estas dinámicas Evolución de la autopercepción del nivel de adquisición de competencias de los estudiantes de una asignatura de Grado en Ingeniería


de evaluación formativa influyen en el nivel de adquisición de las competencias, tanto específicas como transversales, de una asignatura del Grado de Ingeniería Eléctrica.

Palabras clave: Evaluación formativa; adquisición de competencias; metodologías activas

\begin{abstract}
The approach of the education in the university and not university contexts in which we are nowadays makes it necessary to change the methodologies and dynamics developed by teachers in the classroom. This work arises from the necessity of applying active methodologies to the training of university student, and also to the search for functionality and motivation in learning, having the use of instruments and tools with which the student becomes aware of his own teaching-learning process and fosters the critical spirit. The motivation to undertake this practice is raised by the concern to provide the student with skills to help them in their professional development. In the current article it is tried to verify to what extent these formative assessment dynamics influence the level of acquisition of the competences, both specific and transversal, of a subject of the Degree in Electrical Engineering.
\end{abstract}

Keywords: Formative assessment; skills acquisition; active methodologies

\title{
Resumo
}

O foco da educação na universidade e não universitário em que estamos agora necessários mudar metodologias e dinâmicas desenvolvidas por professores nas salas de aula. Este trabalho surge da necessidade de programar metodologias activas (De Miguel Díaz et al., 2006) para a formação de estudantes universitários. Pesquisa funcionalidade e motivação na aprendizagem, fornecendo instrumentos e ferramentas que o aluno tome conhecimento de seu próprio processo de ensino-aprendizagem e estimula o pensamento crítico. A motivação para realizar essa prática é levantada pela preocupação de proporcionar aos alunos habilidades que ajudarão o seu desenvolvimento profissional. Neste artigo é verificar até que ponto você é influência avaliação formativa dinâmica do nível de aquisição de competências, tanto específicas e atravessar uma disciplinana Licenciatura em Engenharia Elétrica.

Palavras-chave: Avaliação formativa; aquisição de competências; metodologias ativas

Evolución de la autopercepción del nivel de adquisición de competencias de los estudiantes de una asignatura de Grado en Ingeniería 


\section{Introducción}

En el contexto de la educación en el que nos encontramos, el gran paradigma al que nos enfrentamos los docentes es pasar de modelos tradicionales en los procesos de enseñanza hacia modelos más abiertos en los que el estudiante forme parte de su propio proceso de aprendizaje (Toom, Husu, \& Patrikainen, 2014). Esto, unido a la necesidad de realizar la evaluación por competencias (Cano, 2008; Spencer \& Spencer, 2008; Zabalza Beraza, 2003) para adaptar nuestras programaciones al Espacio Europeo hace que se desarrollen dinámicas innovadoras dentro del aula (Fernández March, 2005). En este marco se han elaborado modelos de evaluación formativa y compartida (López Pastor, 2009) en asignaturas del ámbito ingenieril. En el presente trabajo se pretende estudiar la incidencia que la metodología de evaluación formativa alcanza sobre el nivel de adquisición de las competencias por parte de los estudiantes. Las experiencias previas a este estudio, arrojaban resultados con un menor grado de concreción, al no contar con un protocolo específico que permitiese instrumentalizar objetivamente los resultados.

\section{Contextualización}

La experiencia se ha realizado con un grupo de estudiantes del Grado de Ingeniería Eléctrica de la Universidad de León en la asignatura de Generación Eléctrica, impartida en el primer semestre del segundo curso. El grupo de estudiantes es reducido (18); esto facilita el desarrollo de una buena práctica. La asignatura es obligatoria de 6 créditos. Se trata de la primera asignatura puramente eléctrica que los estudiantes se encuentran en el Grado. Es una asignatura de carácter eminentemente técnico pero muy generalista, con competencias específicas y transversales imprescindibles para la habilitación de la titulación de Ingeniero Técnico Industrial en la especialidad de Electricidad.

\section{Diseño y desarrollo}

El diseño de la experiencia parte de la inquietud de los docentes por valorar en qué medida contribuyen el desarrollo y presentación de trabajos en grupo a la adquisición de competencias propias de la asignatura. Para ello se realiza un cuestionario de autopercepción, a cumplimentar por los estudiantes al comienzo y al final del cuatrimestre. En dicho cuestionario el estudiante valorará (del 1 al 5, siendo el 1 poco y el 5 excelente) la autopercepción del grado de adquisición de las competencias 
específicas y transversales de la asignatura cursada. Dichas competencias se redactan en forma de subcompetencias para mejor entendimiento y se exponen en la Tabla 1.

La metodología utilizada para el desarrollo de las competencias es la evaluación formativa y compartida (López-Pastor \& Pérez-Pueyo, 2017): se fijan tres momentos a lo largo del cuatrimestre en los cuales se realiza una valoración del trabajo, tanto por parte del profesor (heteroevaluación) como de los estudiantes entre ellos (coevaluación) y hacia ellos (autoevaluación). El desarrollo de esta metodología se ha expuesto en otros trabajos(Gonzalez-Alonso, Fernandez-Diaz, Castejón Limas, \& Pérez-Pueyo, 2016).

Los resultados cualitativos de los cuestionarios inicial y final se exponen en la Tabla 1. Para realizar una comparativa entre inicio y el final en el nivel de logro de las subcompetencias, recurrimos a realizar el promedio de la valoración del total de los estudiantes por subcompetencias, esto es: Promedio adquisición inicial y Promedio adquisición final. Realizando la diferencia de ambos períodos obtenemos la evolución promedio del alumno tipo.

\section{Evaluación}

Obtenidos los valores cualitativos, procedemos a un análisis pormenorizado de la evolución que han tenido los estudiantes en el logro de las competencias. Para una mayor comprensión de los resultados realizamos el gráfico de la Figura 1,que representa visualmente los datos de la Tabla 1. En dicho gráfico se aprecia cómo la autopercepción del desarrollo competencial en el estado final es mayor que el inicial en todas las subcompetencias. Analizando las subcompetencias de forma individual, vemos que la que mayor incremento sufre es la relativa al Conocimiento e identificación de las tecnologías y tipos de Cogeneración, seguida de la concerniente a Conocimiento $e$ identificación de las tecnologías y tipos de Cogeneración. Esto puede ser debido al bajo nivel que inicialmente tienen estas dos competencias, tratándose de temas muy específicos con poca formación en etapas anteriores.

En general, se aprecia un mayor incremento en logro de consecución de las subcompetencias específicas sobre las subcompetencias de tipo transversal. Los perfiles de las gráficas arrojan, asimismo, contornos bien distintos: mientras el perfil del Promedio de adquisición inicial es muy fluctuante; el perfil del Promedio de adquisición final se distingue más homogéneo.

Evolución de la autopercepción del nivel de adquisición de competencias de los estudiantes de una asignatura de Grado en Ingeniería 


\section{Tabla 1.}

Resultados de los cuestionarios inicial y final sobre la autopercepción en la adquisición de las subcompetencias derivadas de las competencias de la asignatura de Generación Eléctrica. Curso

\begin{tabular}{|c|c|c|c|c|}
\hline & Subcompetencias & $\begin{array}{l}\text { Promedio } \\
\text { adquisición } \\
\text { inicial }\end{array}$ & $\begin{array}{l}\text { Promedio } \\
\text { adquisición } \\
\text { final }\end{array}$ & Evolución \\
\hline 1 & Gestión del tiempo & 3,2 & 3,6 & 0,4 \\
\hline 2 & Hábitos de lectura & 2,8 & 3,4 & 0,6 \\
\hline 3 & $\begin{array}{l}\text { Conocimiento sobre aplicaciones informáticas de } \\
\text { apoyo a la asignatura. }\end{array}$ & 2,3 & 2,7 & 0,4 \\
\hline 4 & $\begin{array}{l}\text { Conocimiento sobre técnicas de búsqueda de } \\
\text { información y programas técnicos }\end{array}$ & 3,1 & 3,4 & 0,3 \\
\hline 5 & Estrategias de elaboración de proyectos tecnológicos & 2,6 & 3,0 & 0,4 \\
\hline 6 & Manejo de las TIC como medio expositivo & 2,6 & 3,4 & 0,8 \\
\hline 7 & Habilidad en la exposición oral pública & 2,6 & 3,1 & 0,6 \\
\hline 8 & $\begin{array}{l}\text { Conocimiento del Sistema Eléctrico e Identificación } \\
\text { de sus elementos }\end{array}$ & 3,4 & 4,3 & 0,9 \\
\hline 9 & $\begin{array}{l}\text { Conocimiento e identificación de las centrales } \\
\text { térmicas, tipos, tecnologías, etc. }\end{array}$ & 3,2 & 4,2 & 1,0 \\
\hline 10 & $\begin{array}{l}\text { Conocimiento e identificación de las centrales de } \\
\text { ciclo combinado, tipos, tecnologías, etc. }\end{array}$ & 2,3 & 3,8 & 1,5 \\
\hline 11 & $\begin{array}{l}\text { Conocimiento e identificación de las centrales } \\
\text { nucleares, tipos, tecnologías, etc. }\end{array}$ & 2,9 & 4,1 & 1,2 \\
\hline 12 & $\begin{array}{l}\text { Identificación y diseño de distintos tipos de Centrales } \\
\text { Térmicas }\end{array}$ & 2,4 & 3,6 & 1,1 \\
\hline 13 & $\begin{array}{l}\text { Conocimiento e identificación de las tecnologías de } \\
\text { Generación Eólica. }\end{array}$ & 3,0 & 4,0 & 1,0 \\
\hline 14 & $\begin{array}{l}\text { Conocimiento e identificación de las tecnologías de } \\
\text { Energía solar }\end{array}$ & 3,2 & 3,6 & 0,4 \\
\hline 15 & $\begin{array}{l}\text { Conocimiento e identificación de las tecnologías de } \\
\text { Generación Hidráulica y Minihidráulica. }\end{array}$ & 2,8 & 3,3 & 0,6 \\
\hline 16 & $\begin{array}{l}\text { Conocimiento e identificación de las tecnologías y } \\
\text { tipos de Cogeneración }\end{array}$ & 2,2 & 3,8 & 1,6 \\
\hline 17 & $\begin{array}{l}\text { Identificación y diseño de distintos tipos de Sistemas } \\
\text { de Generación Renovable }\end{array}$ & 2,5 & 3,3 & 0,8 \\
\hline 18 & $\begin{array}{l}\text { Conocimiento de los sistemas de almacenamiento de } \\
\text { energía }\end{array}$ & 2,2 & 3,7 & 1,5 \\
\hline 19 & $\begin{array}{l}\text { Capacidad para el trabajo en equipo, asumiendo roles } \\
\text { y responsabilidades con absoluto respeto a los } \\
\text { derechos fundamentales y a la no discriminación por } \\
\text { razones de sexo, raza, edad o religión }\end{array}$ & 4,3 & 4,4 & 0,1 \\
\hline 20 & $\begin{array}{l}\text { Capacidad para actuar con actitud crítica ante } \\
\text { soluciones ya utilizadas, de manera que le incite a } \\
\text { profundizar en el estudio y análisis de los temas } \\
\text { objeto de esta disciplina. }\end{array}$ & 3,7 & 3,9 & 0,2 \\
\hline 21 & Capacidad para aprender de forma autónoma & 3,7 & 3,8 & 0,1 \\
\hline 22 & $\begin{array}{l}\text { Capacidad para transmitir información, ideas, } \\
\text { problemas y soluciones a un público tanto } \\
\text { especializado como no especializado }\end{array}$ & 3,1 & 3,6 & 0,4 \\
\hline
\end{tabular}

Evolución de la autopercepción del nivel de adquisición de competencias de los estudiantes de una asignatura de Grado en Ingeniería 


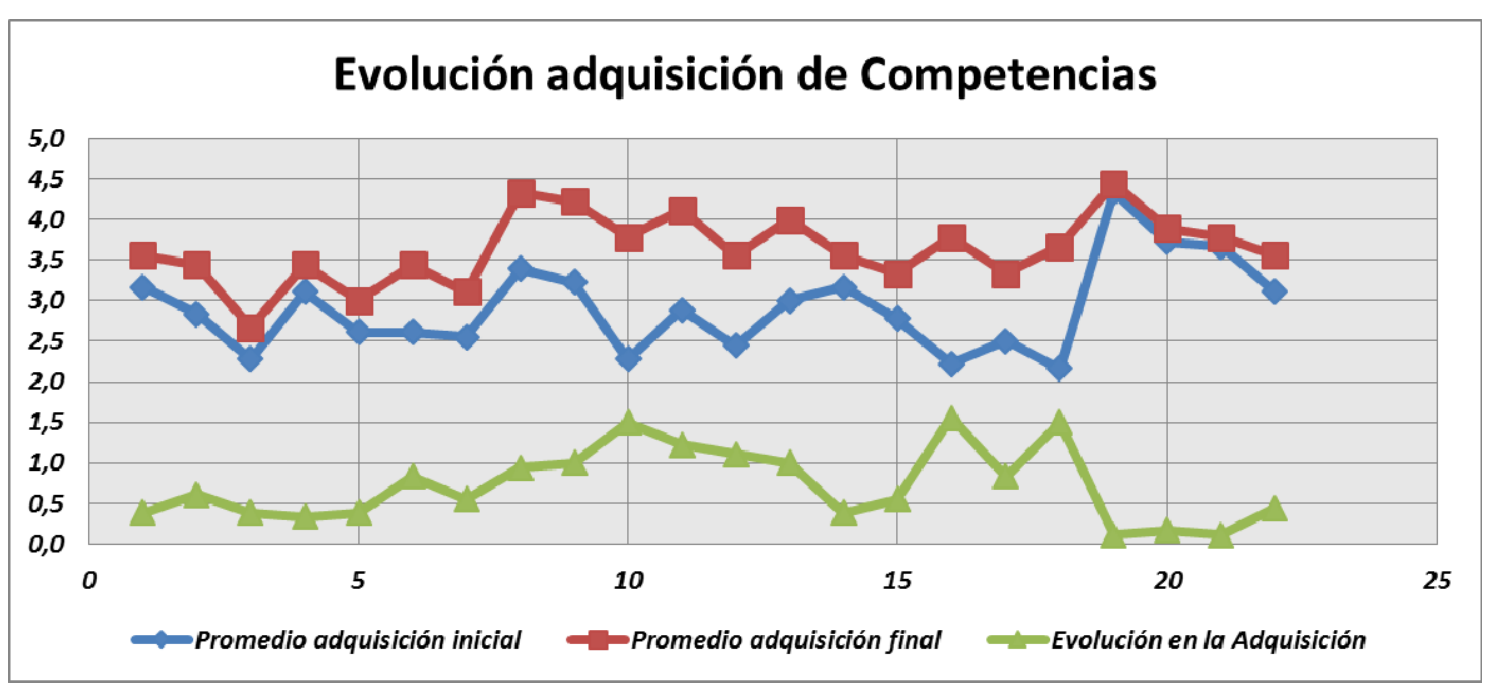

Figura 1.

Gráfico que compara los niveles de competencias adquiridas por los estudiantes antes y después de cursar la asignatura de Generación Eléctrica en el curso 2016/17

\section{Conclusiones}

La principal conclusión obtenida del estudio realizado es que la dinámica propuesta sobre la evaluación formativa establece una clara mejora en la consecución de los objetivos planteados, o sea, en la adquisición del mayor nivel de competencias relativas a la asignatura objeto. Se produce un mayor incremento en aquellas subcompetencias que parten de niveles de adquisición bajos, debido principalmente la carencia de formación previa en dichas subcompetencias (de carácter específico). Por otro lado, las subcompetencias transversales son las que sufren un menor incremento frente a las específicas, cuestión que es de esperar debido precisamente a su transversalidad. La asignatura contribuye a su adquisición de forma conjunta con el resto de asignaturas de la titulación. Además, este tipo de competencias se vienen trabajando en las etapas previas a la universitaria. En todo caso, se constata un incremento generalizado en el logro de competencias que induce a ratificar lo apropiado de las dinámicas propuestas.

Como línea de trabajo futuro se plantea proponer dinámicas activas que contribuyan al incremento del nivel de adquisición de aquellas subcompetencias que no han alcanzado en el presente estudio la media del resto de subcompetencias. En este caso, se encuentran las competencias de Conocimiento sobre aplicaciones informáticas de apoyo a la asignatura, Estrategias de elaboración de proyectos tecnológicos, Habilidad en la exposición oral pública.

Evolución de la autopercepción del nivel de adquisición de competencias de los estudiantes de una asignatura de Grado en Ingeniería 


\section{Referencias}

Cano, E. (2008). La evaluación por competencias en la educación superior. Profesorado: Revista de Currículum Y Formación Del Profesorado, 12(3), 11-27.

De Miguel Díaz, M., Alfaro Rocher, I., Apodaca Urquijo, P., Arias Blanco, J., García Jiménez, E., \& Lobato Fraile, C. (2006). Metodologías de enseñanza y aprendizaje para el desarrollo de competencias: Orientaciones para el profesorado universitario ante el Espacio Europeo de Educación Superior. (M. De Miguel Díaz, Ed.). Madrid: Alianza.

Fernández March, A. (2005). Nuevas metodologías docentes. Talleres de Formación del profesorado para la Convergencia Europea impartidos en la UPM. Valencia: Instituto de Ciencias de la Educación Universidad Politécnica de Valencia.

Gonzalez-Alonso, M.-I., Fernandez-Diaz, R.-A., Castejón Limas, M., \& Pérez-Pueyo, A. (2016). Formative assessment as a tool for acquisition of competences in engineering subjects. EduRe Journal, Vol. 2( $\left.\mathrm{N}^{0} 3\right)$, 79-94. Retrieved from http://edure.org/EduReJournalVol2N3/EduRe_V2_I3_P5.pdf

López-Pastor, V., \& Pérez-Pueyo, Á. (2017). Evaluación formativa y compartida en educación: experiencias de éxito en todas las etapas educativas. Retrieved from http://buleria.unileon.es/handle/10612/5999

López Pastor, V. M. (2009). Evaluación formativa y compartida en Educación Superior: propuestas, técnicas, instrumentos y experiencias. Madrid: Narcea.

Spencer, L. M., \& Spencer, P. S. M. (2008). Competence at Work: Models for Superior Performance. New York: John Wiley \& Sons.

Toom, A., Husu, J., \& Patrikainen, S. (2014). Student teachers’ patterns of reflection in the context of teaching practice. European Journal of Teacher Education, 38(3), 320-340. https://doi.org/10.1080/02619768.2014.943731

Zabalza Beraza, M. Á. (2003). Competencias docentes del profesorado universitario: calidad y desarrollo profesional (Vol. 4). Madrid: Narcea.

Evolución de la autopercepción del nivel de adquisición de competencias de los estudiantes de una asignatura de Grado en Ingeniería 